\title{
Prevalence and correlates of major injuries among older adults in India: Results of a national cross- sectional survey in 2017-2018
}

\author{
Supa Pengpid \\ Mahidol University \\ Karl Peltzer ( $\boldsymbol{D}$ kfpeltzer@gmail.com ) \\ University of the Free State
}

\section{Research Article}

Keywords: health status, health risk behaviour, injury, India

Posted Date: March 2nd, 2021

DOI: https://doi.org/10.21203/rs.3.rs-249948/v1

License: (c) (i) This work is licensed under a Creative Commons Attribution 4.0 International License.

Read Full License 


\section{Abstract}

Background: The study aimed to examine the prevalence and correlates of injury among older adults in India.

Methods: The study included 72,262 individuals (45 years and older) from the cross-sectional 2017-2018 Longitudinal Ageing Study in India (LASI) Wave 1.

Results: More than one in ten participants (12.9\%) had a major injury in the past 2 years, $2.3 \%$ had a road traffic injury, $9.4 \%$ had a fall injury and $1.9 \%$ had other injuries in the past 2 years. In adjusted logistic regression analysis, having 5-9 years education (Adjusted Odds Ratio-AOR: 1.14, 95\% Confidence IntervalCl: 1.01-1.79), having sleep problems (AOR: 1.39, 95\% Cl: 1.23-1.57), having two or more chronic conditions (AOR: 1.21, 95\% Cl: 1.05-1.39), functional disability (AOR: 1.19, 95\% Cl: 1.04-1.35), pain (AOR: 1.81, 95\% Cl: 1.63-2.02), binge drinking (AOR: 1.27, 95\% Cl: 1.03-1.55), tobacco use (AOR: $1.22,95 \% \mathrm{Cl}$ : 1.10-1.36), poor near vision (AOR: 1.17, 95\% Cl: 1.03-1.33), and hearing problems (AOR: $1.30,95 \% \mathrm{Cl}$ : 1.11-1.52) were positively associated with past two years major injury. Compared to Scheduled tribe, Scheduled caste (AOR: 0.65, 95\% Cl: 0.54-0.78), and other backward class (AOR: 0.85, 95\% Cl: 0.76-0.96), having high subjective socioeconomic status (AOR: $0.85,95 \% \mathrm{Cl}$ : 0.75-0.97), and urban residence (AOR: $0.82,95 \% \mathrm{Cl}: 0.73-0.93)$ were negatively associated with past two years major injury. Younger age and male sex were associated with road traffic injury and older age and female sex with were associated with fall injury. Rural residence was associated with fall and other injury. Poor grip strength was positively associated with fall and other injury. Having depressive symptoms and vigorous physical activity were associated with other injury

Conclusion: More than one in ten participants (12.9\%) had a major injury in the past 2 years, and several risk factors were found that can be utilized in injury prevention strategies in India.

\section{Introduction}

"Unintentional injuries are typically classified according to the means of their occurrence: poisoning, burns and scalds, drowning, falls and transport-related." [1]. Low- and middle-income countries, such as in India, experiencing an increase in the older population, rapid motorization, urbanization, and industrialization, which may contribute to an increase of injury [2-5]. Older adults may be more vulnerable to injury because of a reduction in muscle mass, loss of mineral bone density, dietary calcium deficiency, a decrease in physical activity, and increase in functional disability $[6,7]$. To monitor the prevalence and risk factors of unintentional non-fatal injury among older adults is vital to inform intervention strategies [8]. In a national study among older adults ( $\geq 50$ years) in India in 2007, the prevalence of past-year unintentional injury was $9.1 \%$, past-year fall-related injuries $6.6 \%$ [9], past-year road traffic injury $2.4 \%$, and other bodily injury (except falls) $2.6 \%$ [10]. In India, $11 \%$ of all deaths are caused by injury [11]. There is a lack of a more recent national study on the prevalence and correlates of injury among older adults in India. 
In other low- and middle-income countries, among older adults ( $\geq 50$ years), the prevalence of past-year unintentional injury was $5.1 \%$ in China, $5.7 \%$ in Ghana, $4.2 \%$ in Mexico and $1.3 \%$ in South Africa, and the prevalence of past-year fall-related injury was 3.1\% in China, $2.6 \%$ in Ghana, $2.8 \%$ in Mexico and $1.0 \%$ in South Africa [9]. The prevalence of past-year road traffic injury was $1.8 \%$ in China, $1.7 \%$ in Ghana, $1.7 \%$ in Mexico, and $1.8 \%$ in South Africa, and the prevalence of other bodily injury (except falls) was $2.1 \%$ in China, $2.8 \%$ in Ghana, $1.4 \%$ in Mexico and $0.9 \%$ in South Africa [10]. In Indonesia, among older adults ( $\geq$ 50 years), the past 2-years prevalence of fall related injuries was $12.8 \%$ [12], in the Philippines among older adults ( $\geq 60$ years), the prevalence of self-reported injuries was 1.2\% [13], in Ecuador among persons ( $\geq 60$ years) the prevalence of past-year fall-related injury was $11.4 \%$ [14], and in a review on falls among older adults in various regions in India, Joseph et al. [15] found that the prevalence of falls ranged from $26-37 \%$.

Risk factors among older adults for injuries, including fall-related injury, may include lower socioeconomic status, older age, female sex, residing in rural areas, mutimorbidity, pain, low grip strength, poor cognitive functioning, functional disability, pain, imbalance, vision problems, hearing problems, sleeping problems, depressive symptoms, physical inactivity, tobacco use, alcohol use and obesity $[9,10$, $12,15-17]$. The aim of the study was to assess the prevalence and correlates of non-fatal injury among persons aged 45 years and older in India.

\section{Methods}

\section{Sample and procedures}

Cross-sectional data from the nationally representative Longitudinal Ageing Study in India (LASI) Wave 1, 2017-2018 were analyzed (the overall household response rate is $96 \%$, and the overall individual response rate is $87 \%$.) [18]. Interview, physical measurement and biomarker data were collected from individuals aged 45 and above and their spouses, regardless of age, in a household survey. Details of the sampling strategy have been described elsewhere [18]. The study was approved by the Indian Council of Medical Research (ICMR) Ethics Committee and written informed consent was obtained from participants [18].

\section{Measures}

\section{Outcome measure}

Major injury was assessed with the question, "In the past two years, have you sustained any

major injury?" (Yes/No) "What was the cause of that injury? [Multiple answers are allowed]" ("Traffic accident, Struck by person or object, c. Fire, flames, burn, electric Shock, Drowning, Poisoning, Animal attack or bite, Fall, and Others"). [18] We grouped these injury causes into 1) road traffic injuries, 2) fall injuries, and 3) other injury. 
Sociodemographic information included age (years), sex (male, female), education (none, $<5$ years, 5-9 years, and $\geq 10$ years), Cast or tribe (Scheduled tribe, Scheduled caste, other backward classes, none of these), residence (rural, urban) and subjective socioeconomic status. The latter was sourced from the item, "Please imagine a ten-step ladder, where at the bottom are the people who are the worst off - who have the least money, least education, and the worst jobs or no jobs, and at the top of the ladder are the people who are the best off - those who have the most money, most education, and best jobs. Please indicate the number given (1-10) on the rung on the ladder where you would place yourself." [18]. Socioeconomic steps 1 to 3 were classified as poor, $4-5$ as medium, and $5-10$ as high socioeconomic status.

Sleep problems were assessed with four questions: (1) "How often do you have trouble falling asleep?" (2) "How often do you have trouble with waking up during the night?" (3) "How often do you have trouble with waking up too early and not being able to fall asleep again?" (4) "How often do you feel really rested when you wake up in the morning?" Responses options were "never, rarely (1-2 nights per week), occasionally (3-4 nights per week), and frequently (5 or more nights per week)" [18]. Sleep problems were coded as "frequently" for the first three symptoms and "never or rarely" for the fourth symptom as one. Participants who reported any of these four symptoms were classified as having sleep problems [19].

Depressive symptoms were assessed with a modified Centre for Epidemiological Studies Depression Scale (CES-D-10) [20]. The 10 items included seven negative symptoms (trouble concentrating, feeling depressed, low energy, fear of something, feeling alone, bothered by things, and everything is an effort), and three positive symptoms (feeling happy, hopeful, and satisfied). Response options included rarely or never ( $<1$ day), sometimes ( 1 or 2 days), often ( 3 or 4 days), and most or all of the time (5-7 days) in a week prior to the interview. For negative symptoms, rarely or never ( $<1$ day), and sometimes ( 1 or 2 days) were scored zero, and often ( 3 or 4 days) and most or all of the time (5-7 days) categories were scored one. Scoring was reversed for positive symptoms. The overall score ranges from zero to 10 and scores of four or more were indicative for depressive symptoms [21]. The Cronbach a of the CES-D-10 in this study was 0.79 .

Chronic conditions were assessed with the question, "Has any health professional ever told you that you have...?": 1) "Hypertension or high blood pressure (Yes/No); 2) Diabetes or high blood sugar; 3) Cancer or malignant tumor; 4) Chronic lung disease such as asthma, chronic obstructive pulmonary disease/Chronic bronchitis or other chronic lung problems; 5) Chronic heart diseases such as Coronary heart disease (heart attack or Myocardial Infarction), congestive heart failure, or other chronic heart problems; 6) Stroke; 7) Arthritis or rheumatism, Osteoporosis or other bone/joint diseases; 8) Any neurological, or psychiatric problems such as depression, Alzheimer's/Dementia, unipolar/bipolar disorders, convulsions, Parkinson's etc.; and 9) High cholesterol (Yes/No)." [18]. Responses for the nine chronic conditions were summed and trichotomized into 0,1 , or $\geq 2$ chronic conditions.

Functional disability was sourced from Activities of Daily Living (ADL) (6 items) and Instrumental Activities of Daily Living (IADL) (7 items) [22, 23]. Cronbach alpha for the ADL and IADL scale was 0.89 . 
Responses were "Yes/No" and were trichonomized into 0,1, or $\geq 2$ ADL/IADL items.

Hand grip strength (HGS) was measured with a "Baseline Smedley Spring type dynamometer" on "each hand twice, beginning with the dominant hand, alternating hands in between measurements" [18]. A mean HGS (kg) variable was calculated from all four measurements [24]. HGS $<30 \mathrm{~kg}$ for men, $<20 \mathrm{~kg}$ for women using the average value of the four handgrip assessments were defined as "weak" [25].

Symptom-based pain was defined as troubled by pain and required some form of medication or treatment for relief of pain [18].

Anthropometry. "Height and weight of adults were measured using the Seca 803 digital scale." [18]. "BMI was calculated according to Asian criteria: underweight $\left(<18.5 \mathrm{~kg} / \mathrm{m}^{2}\right)$, normal weight $(18.5-22.9$ $\left.\mathrm{kg} / \mathrm{m}^{2}\right)$, overweight $\left(23.0-24.9 \mathrm{~kg} / \mathrm{m}^{2}\right)$, class I obesity $\left(25.0-29.9 \mathrm{~kg} / \mathrm{m}^{2}\right)$, and class II obesity $(\geq 30.0$ $\left.\mathrm{kg} / \mathrm{m}^{2}\right)^{\prime \prime}[26]$.

Binge drinking was assessed with the question, "In the last 3 months, how frequently on average, have you had at least 5 or more drinks on one occasion?" [18] and defined as "one to three days per month, one to four days per week, five or more days per week, or daily."

Current tobacco use was sourced from two items, 1) "Do you currently smoke any tobacco products (cigarettes, bidis, cigars, hookah,cheroot, etc.)? and 2) Do you use smokeless tobacco (such as chewing tobacco, gutka, pan masala, etc.)?" [18].

Vigorous physical activity. " For vigorous activity, respondents were asked about their involvement in running or jogging, swimming, going to a health centre/gym, cycling, digging with a spade or shovel, heavy lifting, chopping, farm work, fast bicycling, and cycling with loads." [18]. Participants, who more than once a week engaged in vigorous physical activity were defined as sufficient physical activity [27].

Vision was assessed with two questions, 1) "How good is your eyesight for seeing things at a distance, like recognizing a person across the street (or 20 meters away), whether or not you wear glasses, contacts, or corrective lenses?" And 2) "How good is your eyesight for seeing things up close, like reading an ordinary newspaper print whether or not you wear glasses, contacts, or corrective lenses?" [18]. Response options ranged from 1 . Very good to 5 . Very poor and poor near or far vision as defined as "poor or very poor"

Cognitive functioning measures in the LASI were derived from the cognition module of the Health and Retirement Study (HRS), including memory (immediate and delayed word recall), orientation (time and place), arithmetic function (backward counting, serial 7, computation), executive function (paper folding and Pentagon drawing), and object naming. A composite cognitive index is created with the lowest 10th percentile as a proxy measure of poor cognitive functioning [18].

Hearing problem was measured with the item. "Have you ever been diagnosed with any hearing or earrelated problem or condition?" (Yes/No) [18]. 
Inadequate balance was defined as $<10$ seconds or no attempt [18].

\section{Data analysis}

Descriptive statistics were used to describe sociodemographic, health, and injury variables. Adjusted logistic regression was used to estimate the prevalence of past 2-years major injuries, road traffic injuries, fall-related injuries and other injury. No multi-collinearity was detected. $P<0.05$ was considered significant, and missing values were discarded. All statistical operations were conducted with STATA software version 13.0 (Stata Corporation, College Station, TX, USA), taking the multi-stage sample design into account.

\section{Results}

\section{Sample and injury prevalence characteristics}

The study sample included 72,262 persons aged 45 years and older (female spouse, any age) from India. Table 1 describes the sample characteristics. More than one in ten participants (12.9\%) had a major injury in the past 2 years, $2.3 \%$ had a road traffic injury, $9.4 \%$ had a fall injury and $1.9 \%$ had other injuries in the past 2 years (see Table 1). 
Table 1

Sample characteristics and prevalence of type of injury

\begin{tabular}{|c|c|c|c|c|c|}
\hline Variable & Sample & $\begin{array}{l}\text { Major } \\
\text { injury }\end{array}$ & $\begin{array}{l}\text { Road traffic } \\
\text { injury }\end{array}$ & $\begin{array}{l}\text { Fall } \\
\text { injury }\end{array}$ & $\begin{array}{l}\text { Other } \\
\text { injury }\end{array}$ \\
\hline & $\%$ & $\%$ & $\%$ & $\%$ & $\%$ \\
\hline All & 72262 & 12.9 & 2.0 & 9.4 & 1.9 \\
\hline Age (years) & \multirow{2}{*}{$\begin{array}{l}40785 \\
(54.1)\end{array}$} & 11.1 & 2.3 & 7.4 & 1.6 \\
\hline 45-59 (female spouse: any age) & & 15.1 & 1.6 & 11.7 & 2.1 \\
\hline$\geq 60$ & $\begin{array}{l}31477 \\
(45.9)\end{array}$ & & & & \\
\hline \multirow{3}{*}{$\begin{array}{l}\text { Sex } \\
\text { Female } \\
\text { Male }\end{array}$} & \multirow{2}{*}{$\begin{array}{l}41685 \\
(58.0)\end{array}$} & 13.1 & 1.2 & 10.5 & 1.6 \\
\hline & & 12.7 & 3.1 & 7.8 & 2.2 \\
\hline & $\begin{array}{l}30577 \\
(42.0)\end{array}$ & & & & \\
\hline \multirow{3}{*}{$\begin{array}{l}\text { Education } \\
\text { No schooling } \\
<5 \text { years }\end{array}$} & \multirow{2}{*}{$\begin{array}{l}33213 \\
(49.5)\end{array}$} & 13.4 & 1.5 & 10.2 & 2.0 \\
\hline & & 14.4 & 2.1 & 10.8 & 2.1 \\
\hline & $\begin{array}{l}8056 \\
(10.8)\end{array}$ & 13.9 & 2.8 & 9.3 & 2.1 \\
\hline \multirow{2}{*}{$\begin{array}{l}5-9 \text { years } \\
\geq 10 \text { years }\end{array}$} & \multirow{2}{*}{$\begin{array}{l}16911 \\
(21.1) \\
14079 \\
(18.5)\end{array}$} & 9.8 & 2.4 & 6.4 & 1.1 \\
\hline & & & & & \\
\hline \multirow{3}{*}{$\begin{array}{l}\text { Cast/tribe } \\
\text { Scheduled tribe } \\
\text { Scheduled caste }\end{array}$} & \multirow{2}{*}{$\begin{array}{l}12047 \\
(19.7)\end{array}$} & 15.0 & 2.4 & 10.9 & 2.1 \\
\hline & & 10.5 & 1.1 & 7.5 & 2.1 \\
\hline & $\begin{array}{l}12509 \\
(8.8)\end{array}$ & 12.3 & 1.9 & 8.9 & 1.7 \\
\hline \multirow{2}{*}{$\begin{array}{l}\text { Other backward class } \\
\text { None of the above }\end{array}$} & \multirow{2}{*}{$\begin{array}{l}27185 \\
(46.7) \\
17893 \\
(24.9)\end{array}$} & 13.5 & 2.2 & 9.8 & 2.0 \\
\hline & & & & & \\
\hline \multirow{3}{*}{$\begin{array}{l}\text { Subjective socioeconomic status } \\
\text { Low }(1-3) \\
\text { Medium (4-5) }\end{array}$} & 23625 & 14.3 & 2.0 & 10.7 & 2.0 \\
\hline & \multirow{2}{*}{$\begin{array}{l}28380 \\
(38.7)\end{array}$} & 12.8 & 2.1 & 9.0 & 2.0 \\
\hline & & 11.0 & 1.7 & 7.9 & 1.5 \\
\hline High $(6-10)$ & $\begin{array}{l}18134 \\
(24.1)\end{array}$ & & & & \\
\hline
\end{tabular}




\begin{tabular}{|c|c|c|c|c|c|}
\hline Variable & Sample & $\begin{array}{l}\text { Major } \\
\text { injury }\end{array}$ & $\begin{array}{l}\text { Road traffic } \\
\text { injury }\end{array}$ & $\begin{array}{l}\text { Fall } \\
\text { injury }\end{array}$ & $\begin{array}{l}\text { Other } \\
\text { injury }\end{array}$ \\
\hline Residence & \multirow{3}{*}{$\begin{array}{l}46539 \\
(68.2) \\
25723 \\
(31.8)\end{array}$} & & 2.0 & 10.1 & 2.3 \\
\hline Rural & & \multirow{2}{*}{10.8} & \multirow{2}{*}{2.0} & \multirow{2}{*}{7.9} & \multirow{2}{*}{1.0} \\
\hline Urban & & & & & \\
\hline Sleep problems & $\begin{array}{l}8367 \\
(12.7)\end{array}$ & 20.0 & 3.2 & 14.8 & 2.7 \\
\hline Depressive symptoms & $\begin{array}{l}17650 \\
(27.6)\end{array}$ & 15.5 & 2.4 & 11.3 & 2.3 \\
\hline Chronic conditions & $\begin{array}{l}39715 \\
(55.0)\end{array}$ & 11.1 & 1.9 & 7.5 & 1.9 \\
\hline 0 & \multirow{2}{*}{$\begin{array}{l}19336 \\
(26.9)\end{array}$} & 14.3 & 2.2 & 10.6 & 1.9 \\
\hline 1 & & \multirow[t]{2}{*}{16.5} & \multirow[t]{2}{*}{1.9} & \multirow[t]{2}{*}{13.3} & \multirow[t]{2}{*}{1.6} \\
\hline 2 or more & $\begin{array}{l}12991 \\
(18.1)\end{array}$ & & & & \\
\hline Functional disability & $\begin{array}{l}46762 \\
(607)\end{array}$ & 10.7 & 2.1 & 7.2 & 1.6 \\
\hline ADL \& IADL = 0 & \multirow{2}{*}{$\begin{array}{l}7425 \\
(10.5)\end{array}$} & \multirow{3}{*}{$\begin{array}{l}12.2 \\
17.9\end{array}$} & \multirow{2}{*}{2.0} & 8.3 & 2.0 \\
\hline ADL \& IADL $=1$ & & & & 14.3 & 2.3 \\
\hline ADL \& IADL = 2 or more & $\begin{array}{l}17700 \\
(28.8)\end{array}$ & & & & \\
\hline $\begin{array}{l}\text { Grip strength (men: }<30 \mathrm{~kg} \text {, } \\
\text { women: }<20 \mathrm{~kg} \text { ) }\end{array}$ & $\begin{array}{l}41981 \\
(69.7)\end{array}$ & 13.4 & 1.7 & 9.9 & 2.1 \\
\hline Symptom-based pain & $\begin{array}{l}19145 \\
(27.7)\end{array}$ & 19.9 & 2.6 & 14.8 & 2.9 \\
\hline Obesity $\left(\geq 25 \mathrm{~kg} / \mathrm{m}^{2}\right.$ ) & $\begin{array}{l}19636 \\
(28.0)\end{array}$ & 12.4 & 2.3 & 9.0 & 1.2 \\
\hline Past month binge drinking & $\begin{array}{l}2600 \\
(2.9)\end{array}$ & 15.3 & 2.7 & 10.9 & 2.2 \\
\hline Current tobacco use & $\begin{array}{l}21071 \\
(30.4)\end{array}$ & 15.0 & 2.5 & 10.5 & 2.4 \\
\hline Vigorous physical activity & $\begin{array}{l}22210 \\
(32.0)\end{array}$ & 12.2 & 2.3 & 8.0 & 2.2 \\
\hline Poor near vision & $\begin{array}{l}11866 \\
(16.8)\end{array}$ & 17.6 & 2.4 & 13.3 & 2.3 \\
\hline Poor distant vision & $\begin{array}{l}9756 \\
(14.2)\end{array}$ & 17.9 & 2.0 & 14.0 & 2.3 \\
\hline
\end{tabular}




\begin{tabular}{|llllll|}
\hline Variable & Sample & $\begin{array}{l}\text { Major } \\
\text { injury }\end{array}$ & $\begin{array}{l}\text { Road traffic } \\
\text { injury }\end{array}$ & $\begin{array}{l}\text { Fall } \\
\text { injury }\end{array}$ & $\begin{array}{c}\text { Other } \\
\text { injury }\end{array}$ \\
\hline Poor cognitive functioning & $\begin{array}{l}5980 \\
(11.3)\end{array}$ & 14.7 & 1.3 & 11.7 & 1.9 \\
\hline Hearing problem & $\begin{array}{l}4874 \\
(6.6)\end{array}$ & 20.6 & 2.4 & 15.8 & 3.0 \\
\hline Balance (no/<10s) & $\begin{array}{l}2129 \\
(3.9)\end{array}$ & 16.0 & 1.3 & 13.8 & 1.1 \\
\hline
\end{tabular}

\section{Cause of injury}

Majority (72.6\%) had a fall, followed by traffic accident (15.4\%), struck by a person or object $(7.6 \%)$, animal attack or bite (4.9\%), fire, flames, burn or electric shock $(1.1 \%)$, and drowing $(0.1 \%)$.

The younger age group (45-59 years) had more traffic injuries than the older age group ( $\geq 60$ years), while the older age had more fall injuries than the younger age group $(p<0.001)$. Women had more often a fall than men and men had more traffic and struck by person or object injuries than women $(p<0.001)$. Traffic injuries were more prevalent in participants residing in urban than rural areas, while struck by a person or object and animal attack or bites were more common in rural than urban areas $(p<0.001)$ (see Table 2). 
Table 2

Cause of major injury in the past 2 years $(\mathrm{N}=8019)$

\begin{tabular}{|c|c|c|c|c|c|c|c|}
\hline Variable & Total & $\begin{array}{l}45-59 \\
\text { years }\end{array}$ & $\begin{array}{l}60 \text { years and } \\
\text { more }\end{array}$ & Male & Female & Urban & Rural \\
\hline & $\mathrm{N}(\%)$ & $\%$ & $\%$ & $\%$ & $\%$ & $\%$ & $\%$ \\
\hline Traffic accident & $\begin{array}{l}1298 \\
(15.4)\end{array}$ & $20.7 *$ & 10.8 & $24.5^{\star}$ & 9.0 & $19.1^{\star}$ & 14.1 \\
\hline $\begin{array}{l}\text { Struck by person or } \\
\text { object }\end{array}$ & $\begin{array}{l}608 \\
(7.6)\end{array}$ & 8.1 & 7.2 & $9.7^{\star}$ & 6.2 & $5.7^{\star}$ & 8.3 \\
\hline $\begin{array}{l}\text { Fire, flames, burn, } \\
\text { electric shock }\end{array}$ & $65(1.1)$ & 1.1 & 1.1 & 1.5 & 0.9 & 1.1 & 1.1 \\
\hline Drowning & $10(0.1)$ & 0.1 & 0.1 & 0.0 & 0.2 & 0.1 & 0.1 \\
\hline Poisoning & $4(0.0)$ & 0.1 & 0.0 & 0.1 & 0.0 & 0.0 & 0.1 \\
\hline Animal attack or bite & $\begin{array}{l}371 \\
(4.9)\end{array}$ & 5.0 & 4.9 & 5.4 & 4.6 & $1.7^{\star}$ & 6.1 \\
\hline Fall & $\begin{array}{l}5782 \\
(72.6)\end{array}$ & $66.7 *$ & 77.8 & $61.3^{\star}$ & 80.6 & 73.5 & 72.3 \\
\hline Other & $66(0.7)$ & 0.6 & 0.8 & 0.8 & 0.7 & 0.3 & 0.9 \\
\hline${ }^{*} p<0.001$ & & & & & & & \\
\hline
\end{tabular}

\section{Associations with injury types}

In adjusted logistic regression analysis, having 5-9 years education (Adjusted Odds Ratio-AOR: 1.14, 95\% Confidence Interval-Cl: 1.01-1.79), having sleep problems (AOR: 1.39, 95\% Cl: 1.23-1.57), having two or more chronic conditions (AOR: 1.21, 95\% Cl: 1.05-1.39), functional disability (AOR: 1.19, 95\% Cl: 1.041.35), pain (AOR: 1.81, 95\% Cl: 1.63-2.02),, binge drinking (AOR: 1.27, 95\% Cl: 1.03-1.55), tobacco use (AOR: 1.22 , 95\% Cl: 1.10-1.36), poor near vision (AOR: 1.17, 95\% Cl: 1.03-1.33), and hearing problems (AOR: $1.30,95 \% \mathrm{Cl}: 1.11-1.52$ ) were positively associated with past two years major injury. Compared to Scheduled tribes, Scheduled castes (AOR: $0.65,95 \% \mathrm{Cl}: 0.54-0.78$ ), and other backward classes (AOR: $0.85,95 \% \mathrm{Cl}: 0.76-0.96)$, having high subjective socioeconomic status (AOR: 0.85, 95\% Cl: 0.75-0.97), and urban residence (AOR: $0.82,95 \% \mathrm{Cl}$ : 0.73-0.93) were negatively associated with past two years major injury. Younger age and male sex were associated with road traffic injury, and older age and female sex with were associated with fall injury. Rural residence was associate with fall and other injuries. Poor grip strength was positively associated with fall and other injuries. Having depressive symptoms and vigorous physical activity were associated with other injuries (see Table 3 ). 
Table 3

Associations with injury

\begin{tabular}{|c|c|c|c|c|}
\hline Variable & Major injury & $\begin{array}{l}\text { Road traffic } \\
\text { injury }\end{array}$ & Fall injury & Other injury \\
\hline & AOR (95\% Cl) & AOR $(95 \% \mathrm{Cl})$ & AOR $(95 \% \mathrm{Cl})$ & $\operatorname{AOR}(95 \% \mathrm{Cl})$ \\
\hline Age (years) & 1 (Reference) & 1 (Reference) & 1 (Reference) & 1 (Reference) \\
\hline $\begin{array}{l}45-59 \text { (female spouse: any } \\
\text { age) }\end{array}$ & $\begin{array}{l}1.10(0.98 \\
1.23)\end{array}$ & $\begin{array}{l}0.68(0.52 \\
0.90)^{\star \star}\end{array}$ & $\begin{array}{l}1.23(1.08 \\
1.41^{\star \star}\end{array}$ & $\begin{array}{l}1.11(0.91 \\
1.37)\end{array}$ \\
\hline \multicolumn{5}{|l|}{$\geq 60$} \\
\hline Sex & 1 (Reference) & 1 (Reference) & 1 (Reference) & 1 (Reference) \\
\hline Female & $\begin{array}{l}0.99(0.88 \\
1.12)\end{array}$ & $\begin{array}{l}3.04(2.29 \\
4.04)^{\star \star \star}\end{array}$ & $\begin{array}{l}0.70(0.60 \\
0.80)^{\star \star \star *}\end{array}$ & $\begin{array}{l}1.32(1.00 \\
1.74)\end{array}$ \\
\hline \multicolumn{5}{|l|}{ Male } \\
\hline Education & 1 (Reference) & 1 (Reference) & 1 (Reference) & 1 (Reference) \\
\hline No schooling & $\begin{array}{l}1.05(0.90 \\
1.23)\end{array}$ & $\begin{array}{l}1.05(0.74 \\
1.48)\end{array}$ & $\begin{array}{l}1.14(0.95 \\
1.37)\end{array}$ & $\begin{array}{l}0.89(0.66 \\
1.20)\end{array}$ \\
\hline $\begin{array}{l}<5 \text { years } \\
5-9 \text { years }\end{array}$ & $\begin{array}{l}1.14(1.01 \\
1.29)^{\star}\end{array}$ & $\begin{array}{l}1.35(1.01 \\
1.79)^{\star}\end{array}$ & $\begin{array}{l}1.09(0.95 \\
1.26)\end{array}$ & $\begin{array}{l}1.10(0.82 \\
1.49)\end{array}$ \\
\hline$\geq 10$ years & $\begin{array}{l}0.88(0.75 \\
1.05)\end{array}$ & $\begin{array}{l}1.10(0.81 \\
1.50)\end{array}$ & $\begin{array}{l}0.85(0.68 \\
1.07)\end{array}$ & $\begin{array}{l}0.72(0.52 \\
1.00)\end{array}$ \\
\hline Cast/tribe & 1 (Reference) & 1 (Reference) & 1 (Reference) & 1 (Reference) \\
\hline Scheduled tribe & $\begin{array}{l}0.65(0.54 \\
0.78)^{\star \star \star \star}\end{array}$ & $\begin{array}{l}0.48(0.32 \\
0.73)^{\star \star \star}\end{array}$ & $\begin{array}{l}0.67(0.54 \\
0.84)^{\star \star \star \star}\end{array}$ & $\begin{array}{l}0.88(0.58 \\
1.35)\end{array}$ \\
\hline Other backward class & $\begin{array}{l}0.85(0.76 \\
0.96)^{\star \star}\end{array}$ & $\begin{array}{l}0.77(0.57 \\
1.05)\end{array}$ & $\begin{array}{l}0.86(0.76 \\
0.99)^{\star}\end{array}$ & $\begin{array}{l}0.93(0.72 \\
1.20)\end{array}$ \\
\hline None of the above & $\begin{array}{l}0.98(0.87 \\
1.12)\end{array}$ & $\begin{array}{l}0.91(0.67 \\
1.25)\end{array}$ & $\begin{array}{l}0.98(0.84, \\
1.11)\end{array}$ & $\begin{array}{l}1.33(1.01 \\
1.73)^{\star}\end{array}$ \\
\hline \multirow{2}{*}{$\begin{array}{l}\text { Subjective socioeconomic } \\
\text { status }\end{array}$} & 1 (Reference) & 1 (Reference) & 1 (Reference) & 1 (Reference) \\
\hline & $\begin{array}{l}0.99(0.89 \\
1.10)\end{array}$ & $\begin{array}{l}1.03(0.79 \\
1.33)\end{array}$ & $\begin{array}{l}0.92(0.82 \\
1.04)\end{array}$ & $\begin{array}{l}1.25(0.99 \\
1.57)\end{array}$ \\
\hline Medium (4-5) & \multirow{2}{*}{$\begin{array}{l}0.85(0.75 \\
0.97)^{\star}\end{array}$} & \multirow{2}{*}{$\begin{array}{l}0.78(0.59 \\
1.02)\end{array}$} & \multirow{2}{*}{$\begin{array}{l}0.82(0.70 \\
0.96)^{\star}\end{array}$} & \multirow{2}{*}{$\begin{array}{l}1.12(0.88 \\
1.44)\end{array}$} \\
\hline High $(6-10)$ & & & & \\
\hline Residence & 1 (Reference) & 1 (Reference) & 1 (Reference) & 1 (Reference) \\
\hline Rural & \multirow[t]{2}{*}{$\begin{array}{l}0.82(0.73 \\
0.93)^{\star \star}\end{array}$} & \multirow[t]{2}{*}{$\begin{array}{l}1.06(0.79 \\
1.42)\end{array}$} & \multirow[t]{2}{*}{$\begin{array}{l}0.81(0.70 \\
0.93)^{\star \star}\end{array}$} & \multirow[t]{2}{*}{$\begin{array}{l}0.61(0.47 \\
0.80)^{\star \star \star}\end{array}$} \\
\hline Urban & & & & \\
\hline
\end{tabular}

AOR = Adjusted Odds Ratio; $\mathrm{Cl}=$ Confidence Interval; ${ }^{* \star *} p<0.001 ;{ }^{* \star} p<0.01 ;{ }^{*} p<0.05$ 


\begin{tabular}{|c|c|c|c|c|}
\hline Variable & Major injury & $\begin{array}{l}\text { Road traffic } \\
\text { injury }\end{array}$ & Fall injury & Other injury \\
\hline Sleep problems & $\begin{array}{l}1.39(1.23 \\
1.57)^{\star \star \star \star}\end{array}$ & $\begin{array}{l}1.71(1.31 \\
2.22)^{\star \star \star}\end{array}$ & $\begin{array}{l}1.33(1.16 \\
1.53)^{\star \star \star}\end{array}$ & $\begin{array}{l}1.20(0.93 \\
1.56)\end{array}$ \\
\hline Depressive symptoms & $\begin{array}{l}1.12(1.00 \\
1.24)\end{array}$ & $\begin{array}{l}1.33(0.96 \\
1.83)\end{array}$ & $\begin{array}{l}1.03(0.92 \\
1.15)\end{array}$ & $\begin{array}{l}1.35(1.09 \\
1.67)^{\star *}\end{array}$ \\
\hline Chronic conditions & 1 (Reference) & 1 (Reference) & 1 (Reference) & 1 (Reference) \\
\hline 0 & $\begin{array}{l}1.19(1.06 \\
1.33)^{\star \star}\end{array}$ & $\begin{array}{l}1.13(0.83 \\
1.54)\end{array}$ & $\begin{array}{l}1.23(1.09 \\
1.40)^{\star \star \star}\end{array}$ & $\begin{array}{l}1.07(0.83 \\
1.39)\end{array}$ \\
\hline 2 or more & $\begin{array}{l}1.21(1.05 \\
1.39)^{\star \star}\end{array}$ & $\begin{array}{l}0.94(0.70 \\
1.26)\end{array}$ & $\begin{array}{l}1.37(1.16 \\
1.62)^{\star \star \star}\end{array}$ & $\begin{array}{l}0.89(0.68 \\
1.16)\end{array}$ \\
\hline Functional disability & 1 (Reference) & 1 (Reference) & 1 (Reference) & 1 (Reference) \\
\hline ADL \& IADL = 0 & $\begin{array}{l}0.94(0.82, \\
1.09)\end{array}$ & $\begin{array}{l}0.91(0.65 \\
1.29)\end{array}$ & $\begin{array}{l}0.94(0.79 \\
1.11)\end{array}$ & $\begin{array}{l}0.93(0.70 \\
1.25)\end{array}$ \\
\hline$A D L \& I A D L=2$ or more & $\begin{array}{l}1.19(1.04 \\
1.35)^{\star \star}\end{array}$ & $\begin{array}{l}0.92(0.71 \\
1.19)\end{array}$ & $\begin{array}{l}1.22(1.05 \\
1.42)^{\star \star}\end{array}$ & $\begin{array}{l}1.25(0.92 \\
1.70)\end{array}$ \\
\hline $\begin{array}{l}\text { Grip strength (men: }<30 \mathrm{~kg} \text {, } \\
\text { women: }<20 \mathrm{~kg} \text { ) }\end{array}$ & $\begin{array}{l}1.05(0.94 \\
1.17)\end{array}$ & $\begin{array}{l}0.71(0.55 \\
0.92)^{\star \star}\end{array}$ & $\begin{array}{l}1.14(1.00 \\
1.29)^{\star}\end{array}$ & $\begin{array}{l}1.27(1.03 \\
1.57)^{\star}\end{array}$ \\
\hline Symptom-based pain & $\begin{array}{l}1.81(1.63 \\
2.02)^{\star \star \star}\end{array}$ & $\begin{array}{l}1.59(1.28 \\
1.97)^{\star \star \star}\end{array}$ & $\begin{array}{l}1.75(1.54 \\
1.98)^{\star \star \star}\end{array}$ & $\begin{array}{l}1.85(1.45 \\
2.32)^{\star \star \star}\end{array}$ \\
\hline Obesity ( $\left.\geq 25 \mathrm{~kg} / \mathrm{m}^{2}\right)$ & $\begin{array}{l}0.99(0.87 \\
1.11)\end{array}$ & $\begin{array}{l}1.26(0.94 \\
1.71)\end{array}$ & $\begin{array}{l}0.97(0.84 \\
1.11)\end{array}$ & $\begin{array}{l}0.66(0.51 \\
0.85)^{\star \star \star}\end{array}$ \\
\hline Past month binge drinking & $\begin{array}{l}1.27(1.03 \\
1.55)^{\star}\end{array}$ & $\begin{array}{l}0.90(0.61 \\
1.34)\end{array}$ & $\begin{array}{l}1.51(1.20 \\
1.89)^{\star \star \star}\end{array}$ & $\begin{array}{l}0.88(0.49 \\
1.59)\end{array}$ \\
\hline Current tobacco use & $\begin{array}{l}1.22(1.10 \\
1.36)^{\star \star \star}\end{array}$ & $\begin{array}{l}0.92(0.68 \\
1.24)\end{array}$ & $\begin{array}{l}1.33(1.18 \\
1.50)^{\star \star \star}\end{array}$ & $\begin{array}{l}1.08(0.84 \\
1.38)\end{array}$ \\
\hline Vigorous physical activity & $\begin{array}{l}1.08(0.97 \\
1.22)\end{array}$ & $\begin{array}{l}1.02(0.81 \\
1.30)\end{array}$ & $\begin{array}{l}1.03(0.89 \\
1.19)\end{array}$ & $\begin{array}{l}1.35(1.09 \\
1.67)^{\star \star}\end{array}$ \\
\hline Poor near vision & $\begin{array}{l}1.17(1.03 \\
1.33)^{\star}\end{array}$ & $\begin{array}{l}1.25(0.86 \\
1.81)\end{array}$ & $\begin{array}{l}1.16(1.01 \\
1.34)^{\star}\end{array}$ & $\begin{array}{l}0.90(0.70 \\
1.15)\end{array}$ \\
\hline Poor distant vision & $\begin{array}{l}1.12(0.98 \\
1.29)\end{array}$ & $\begin{array}{l}0.89(0.62 \\
1.28)\end{array}$ & $\begin{array}{l}1.15(0.99 \\
1.35)\end{array}$ & $\begin{array}{l}1.12(0.85 \\
1.48)\end{array}$ \\
\hline Poor cognitive functioning & $\begin{array}{l}0.91(0.78 \\
1.05)\end{array}$ & $\begin{array}{l}0.98(0.60 \\
1.59)\end{array}$ & $\begin{array}{l}0.89(0.75 \\
1.05)\end{array}$ & $\begin{array}{l}0.79(0.58 \\
1.08)\end{array}$ \\
\hline Hearing problem & $\begin{array}{l}1.30(1.11 \\
1.52)^{\star \star \star}\end{array}$ & $\begin{array}{l}1.14(0.75 \\
1.74)\end{array}$ & $\begin{array}{l}1.30(1.08 \\
1.56)^{\star \star}\end{array}$ & $\begin{array}{l}1.53(1.12 \\
2.09)^{\star \star}\end{array}$ \\
\hline
\end{tabular}

AOR = Adjusted Odds Ratio; $\mathrm{Cl}=$ Confidence Interval; ${ }^{* \star \star} p<0.001 ;{ }^{* \star} p<0.01 ;{ }^{\star} p<0.05$ 


\begin{tabular}{|c|c|c|c|c|}
\hline Variable & Major injury & $\begin{array}{l}\text { Road traffic } \\
\text { injury }\end{array}$ & Fall injury & Other injury \\
\hline Balance $(\mathrm{no} /<10 \mathrm{~s})$ & $\begin{array}{l}0.86(0.66 \\
1.11)\end{array}$ & $\begin{array}{l}0.71(0.29 \\
1.77)\end{array}$ & $\begin{array}{l}0.91(0.69, \\
1.21)\end{array}$ & $\begin{array}{l}0.67(0.39 \\
1.16)\end{array}$ \\
\hline
\end{tabular}

\section{Discussion}

The study found in a national population-based survey among older adults ( $\geq 45$ years) in India in 20172018 , a prevalence of $12.9 \%$ major injuries in the past 2 years, $2.3 \%$ road traffic injury, $9.4 \%$ had a fall injury and $1.9 \%$ had other injuries in the past 2 years, which are similar to past-year prevalence rates of $9.1 \%$ injury, $6.6 \%$ fall-related injury, $2.4 \%$ road traffic injuries, and other bodily injury (except falls) in the India 2007 national survey among older adults ( $\geq 50$ years) $[9,10]$. The prevalence of injury $(12.9 \%$ past 3 years) in this study seems higher than in China (5.1\% past year), $5.7 \%$ in Ghana (5.7\% past year), $4.2 \%$ in Mexico (4.2\%) and 1.3\% in South Africa (1.3\%) [9], while the prevalence of fall-related injury (9.4\%) is higher than in China (3.1\%), Ghana (2.6\%), Mexico (2.8\%) and South Africa (1.0\%) [9], but lower than in Indonesia (12.8\% past 2 years) [12], and Ecuador (11.4\% past year) [14]. The prevalence of road traffic injury $(2.3 \%)$ and other injuries $(1.9 \%)$ in the past 2 years in this study were similar to previous studies, ranging from $1.7 \%$ in China and Mexico to $1.8 \%$ in China and South Africa in terms of road traffic injuries (past year) and ranging from $0.9 \%$ in South Africa to $2.8 \%$ in Ghana in terms of other injury $[9,10]$.

In line with previous research $[4,10]$, the most common non-road traffic injuries in this study were falls, struck by a person or object and animal attacks or bite. The latter two were significantly more common in rural areas in this study. The high proportion of interpersonal violence (struck by a person or objects) was also found in the previous SAGE study among older adults [10], which should be addressed in interventions to reduce injuries in older adults [10]. As found in the six SAGE countries study [9], the majority of injuries were fall-related, confirming that the most common cause of injury among adults in falls [28].

Among older women the prevalence of fall injuries was higher than in younger men, which is consistent with previous findings [9, 29-32]. Among younger men and those living in urban areas, the prevalence of road traffic injuries was higher than in younger women and those living in rural areas in this study. In a multi-country study among persons aged 35-70 years younger age, males and in low-income countries, urban residence were associated with road traffic injuries [32]. In a study on road traffic injury in a tertiary care hospital in India, urban residence also outnumbered rural residents [33]. The gender differences in terms of higher fall injury rates among women than men may be attributed to "differences in higher levels of physical activity, muscle strength, bone density, and fatal fall rates in men than in women" [34]. In terms of gender differences regarding road traffic injury, this may be related to higher risk-taking behaviour among men than women [35]. Fall injuries were significantly higher in rural than urban areas in 
this study. It is possible that environmental factors such as "open street gutters, poor quality footpaths, and unsafe walking areas" in rural areas contribute to this $[9,36]$.

In agreement with previous research $(9,27,38)$, higher socioeconomic status reduced the odds of injury, including fall injuries.

In relation to the different caste groups, compared to members of the Scheduled tribe, members of the Scheduled castes and other backward class had lower odds of injury, including fall injury. Among all caste groups, Schedule tribe had the lowest income, followed by Scheduled castes and other backward classes [18]. Compared to other backward classes, Scheduled castes and Schedule tribes have experienced the largest deprivation within the Indian social hierarchy [39]. This seems to partially confirm that castes at the higher social hierarchy and higher economic status have decreased odds of injury. Regarding educational status, compared to persons with no formal schooling, persons with 5 to 9 years education had a higher likelihood of having had a major injury, including road traffic injuries.

In agreement with previous research [9, 10, 16, 17, 40-43], pain and sleeping problems were associated with both road traffic and fall injury. Sleep problems may increase drowsiness, increasing the risk of road traffic and fall injuries $[42,43]$. To target interventions, further research should establish the type of sleep problems that increase the risk of injury [10]. While some studies $[9,12,44]$ found that depressive symptoms were associated with injurous falls and unintentional injury in general, this study only found a positive association between depressive symptoms and other injuries.

In line with previous research $[9,12,37,38,46,47]$, this study found an association between functional disability, visual difficulties, hearing problems, and injurious falls and/or injury in general. In addition, multimorbidity was found associated with fall injuries, as found in previous studies $[9,12,44]$. Having functional disability and multimorbidity may compromise one's mobility, increasing the risk of falls [12]. Vision problems and/or hearing problems may increase the risk for falling because of poor perception of spatial distances and relationships [48, 49]. Unlike some former findings [44], this study did not find a significant association between inadequate standing balance and injury, including fall-related injury.

In agreement with previous research [50], low hand grip strength was positively associated with fall and other injuries. While some studies showed an association between poor cognitive functioning $[9,12,37]$ and injury, we did not find any significant association with any injury indicator. In this study, heavy episodic drinking and current tobacco use were associated with injury in general, including fall injury but not road traffic injury. In a study among emergency department patients in India alcohol was found to be involved in various types of injuries, including road traffic injuries [51]. Vigorous physical activity increased the odds of other injury in this study, while among middle-aged and older adults in the USA, leisure-time physical activity increased the risk of fall injuries [52]. In a study in a high-income setting (Texas, USA) [53], obesity increased the odds of fall injury among persons 45 years and older, while in this study it was not fall but other injuries.

Study limitations include the cross-sectional design and self-report of the data. The reference period for injury was the past two years, which may have introduced a recall bias. Some variables were not 
assessed, such as the impact of injuries, which should be included in future research.

\section{Conclusions}

Among older adults in India, more than one in ten participants (12.9\%) had a major injury in the past 2 years, and several risk factors, such as lower subjective socioeconomic status, Scheduled tribe, rural residents, sleep problems, chronic conditions, functional disability, pain, heavy episodic drinking current tobacco use, poor near vision and hearing problems, were identified that help guide injury prevention efforts in India.

\section{Declarations}

All methods were carried out in accordance with relevant guidelines and regulations.

\section{Ethics approval and consent to participate}

The study was approved by the Indian Council of Medical Research (ICMR) Ethics Committee and written informed consent was obtained from participants.

\section{Consent for publication}

Not applicable

\section{Availability of data and materials}

The data are available at the The Gateway to Global Aging Data (www.g2aging.org).

\section{Competing interests}

The authors declare that they have no competing interests.

\section{Funding}

The analysis received no funding

\section{Authors' contributions}

"All authors fulfil the criteria for authorship. SP and KP conceived and designed the research, performed statistical analysis, drafted the manuscript, and made critical revisions of the manuscript for key intellectual content. All authors read and approved the final version of the manuscript and have agreed to the authorship and order of authorship for this manuscript."

\section{Acknowledgements}

"The Longitudinal Aging Study in India Project is funded by the Ministry of Health and Family Welfare, Government of India, the National Institute on Aging (R01 AG042778, R01 AG030153), and United 
Nations Population Fund, India."

\section{References}

1. World Health Organization (WHO) (2010). Injuries and violence: the facts. Geneva, Switzerland: WHO.

2. Chandran, A., Hyder, A. A., \& Peek-Asa, C. (2010). The global burden of unintentional injuries and an agenda for progress. Epidemiologic Reviews, 32, 110-120.

3. Nakahara, S., \& Ichikawa, M. (2006). Injuries are growing public health concern in low- and middleincome countries (LMICs). Journal of Trauma, 61, 768.

4. Moncatar TJR, Nakamura K, Siongco KL, Rahman M, Seino K. Prevalence and Determinants of SelfReported Injuries among Community-Dwelling Older Adults in the Philippines: A 10-Year Pooled Analysis. Int J Environ Res Public Health. 2020 Jun 18;17(12):4372. doi: 10.3390/ijerph17124372.

5. Gururaj G. Injuries in India: A national perspective. URL: https://nimhans.ac.in/wpcontent/uploads/2019/02/BD_7-India-Injury-Report_0.pdf (accessed 2 Febr 2021)

6. India State-Level Disease Burden Initiative Road Injury Collaborators. Mortality due to road injuries in the states of India: the Global Burden of Disease Study 1990-2017. Lancet Public Health. 2020 Feb;5(2):e86-e98. doi: 10.1016/S2468-2667(19)30246-4.

7. Navaratnarajah A, Jackson SHD. The physiology of ageing. Medicine in Older Adults. 2017;45(1):610. DOI:https://doi.org/10.1016/j.mpmed.2016.10.008

8. Udofia EA, Aheto JM, Mensah G, Biritwum R, Yawson AE. Prevalence and risk factors associated with non-traffic related injury in the older population in Ghana: Wave 2 of the WHO Study on Global AGEing and adult health (SAGE). Prev Med Rep. 2019 Jun 28;15:100934. doi:

10.1016/j.pmedr.2019.100934. PMID: 31333998; PMCID: PMC6617348.

9. Jagnoor J, Ponnaiah M, Varghese M, Ivers R, Kumar R, Prinja S, Christou A, Jain T. Potential for establishing an injury surveillance system in India: a review of data sources and reporting systems. BMC Public Health. 2020 Dec 14;20(1):1909. doi: 10.1186/s12889-020-09992-9.

10. Stewart Williams J, Kowal P, Hestekin H, O'Driscoll T, Peltzer K, Yawson A, Biritwum R, Maximova T, Salinas Rodríguez A, Manrique Espinoza B, Wu F, Arokiasamy P, Chatterji S; SAGE collaborators. Prevalence, risk factors and disability associated with fall-related injury in older adults in low- and middle-incomecountries: results from the WHO Study on global AGEing and adult health (SAGE). BMC Med. 2015 Jun 23;13:147. doi: 10.1186/s12916-015-0390-8.

11. Peltzer, K., Phaswana-Mafuya, N., Arokiasamy, P., Biritwum, R., Yawson, A., Minicuci, N., Stewart Williams, J., Kowal, P., Chatterji, S. \& the SAGE Collaborating Group (2015) Prevalence, circumstances and consequences of non-fatal road traffic injuries and other bodily injuries among older people in China, Ghana, India, Mexico, Russia and South Africa. African Safety Promotion: A Journal of Injury and Violence Prevention, 13(2), 59-77.

12. World Health Organization - Noncommunicable Diseases (NCD) Country Profiles,India, 2018. URL: https://www.who.int/nmh/countries/2018/ind_en.pdf?ua=1 (accessed 5 Febr 2021) 
13. Pengpid S, Peltzer K. Prevalence and Risk Factors Associated with Injurious Falls among Community-Dwelling Older Adults in Indonesia. Curr Gerontol Geriatr Res. 2018 Jun 3;2018:5964305. doi: $10.1155 / 2018 / 5964305$.

14. Orces $\mathrm{CH}$. Prevalence and Determinants of Fall-Related Injuries among Older Adults in Ecuador. Curr Gerontol Geriatr Res. 2014;2014:863473. doi: 10.1155/2014/863473.

15. Joseph A, Kumar D, Bagavandas M. A Review of Epidemiology of Fall among Elderly in India. Indian J Community Med. 2019 Apr-Jun;44(2):166-168. doi: 10.4103/ijcm.IJCM_201_18.

16. Almegbel FY, Alotaibi IM, Alhusain FA, Masuadi EM, Al Sulami SL, Aloushan AF, Almuqbil BI. Period prevalence, risk factors and consequent injuries of falling among the Saudi elderly living in Riyadh, Saudi Arabia: a cross-sectional study. BMJ Open. 2018 Jan 10;8(1):e019063. doi: 10.1136/bmjopen2017-019063.

17. Bekibele CO, Gureje O. Fall incidence in a population of elderly persons in Nigeria. Gerontology. 2010;56(3):278-83. doi: 10.1159/000236327.

18. International Institute for Population Sciences (IIPS), NPHCE, MoHFW, Harvard T. H. Chan School of Public Health (HSPH) and the University of Southern California (USC) 2020. Longitudinal Ageing Study in India (LASI) Wave 1, 2017-18, India Report, International Institute for Population Sciences, Mumbai.

19. Cho E, Chen TY. The bidirectional relationships between effort-reward imbalance and sleep problems among older workers. Sleep Health. 2020 Jun;6(3):299-305. doi: 10.1016/j.sleh.2020.01.008.

20. Andresen, E.M., Malmgren, J.A., Carter, W.B., Patrick, D.L., 1994. Screening for depression in well older adults: evaluation of a short form of the CES-D (Center for Epidemiologic Studies Depression Scale). Am. J. Prev. Med. 10, 77-84.

21. Kumar S, Nakulan A, Thoppil SP, Parassery RP, Kunnukattil SS. Screening for Depression among Community-dwelling Elders: Usefulness of the Center for Epidemiologic Studies Depression Scale. Indian J Psychol Med. 2016 Sep-Oct;38(5):483-485. doi: 10.4103/0253-7176.191380.

22. Katz S, Ford AB, Moskowitz RW, Jackson BA, Jaffe MW. Studies of illness in the aged. the index of adl: a standardized measure of biological and psychosocial function. JAMA. 1963 Sep 21;185:914-9. doi: 10.1001/jama.1963.03060120024016.

23. Lawton MP, Brody EM. Assessment of older people: self-maintaining and instrumental activities of daily living. Gerontologist. 1969 Autumn;9(3):179-86.

24. Roberts HC, Denison HJ, Martin HJ, Patel HP, Syddall H, Cooper C, Sayer AA. A review of the measurement of grip strength in clinical and epidemiological studies: towards a standardised approach. Age Ageing. 2011 Jul;40(4):423-9. doi: 10.1093/ageing/afr051.

25. Cruz-Jentoft, A.J.; Baeyens, J.P.; Bauer, J.M.; Boirie, Y.; Cederholm, T.; Landi, F.; Martin, F.C.; Michel, J.P.; Rolland, Y.; Schneider, S.M.; et al. Sarcopenia: European consensus on definition and diagnosis: Report of the European Working Group on Sarcopenia in Older People. Age Ageing 2010, 39, 412423. 
26. Wen CP, David Cheng TY, Tsai SP, Chan HT, Hsu HL, Hsu CC, Eriksen MP. Are Asians at greater mortality risks for being overweight than Caucasians? Redefining obesity for Asians. Public Health Nutr. 2009 Apr;12(4):497-506. doi: 10.1017/S1368980008002802.

27. Lübs L, Peplies J, Drell C, Bammann K. Cross-sectional and longitudinal factors influencing physical activity of 65 to 75-year-olds: a pan European cohort study based on the survey of health, ageing and retirement in Europe (SHARE). BMC Geriatr. 2018 Apr 16;18(1):94. doi: 10.1186/s12877-018-0781-8.

28. Karlsson MK, Vonschewelov T, Karlsson C, Cöster M, Rosengen BE. Prevention of falls in the elderly: a review. Scand J Public Health. 2013 Jul;41(5):442-54. doi: 10.1177/1403494813483215.

29. Chan KM, Pang WS, Ee $\mathrm{CH}$, et al. Epidemiology of falls among the elderly community dwellers in Singapore. Singapore Med J. 1997;38(10):427-31

30. D'souza SA, Shringarpure A, Karol J. Circumstances and consequences of falls in Indian older adults. Indian J Occup Therapy. 2008;40(1):3-11.

31. Mitchell RJ, Watson WL, Milat A, et al. Health and lifestyle risk factors for falls in a large populationbased sample of older people in Australia. J Safety Res. 2013;45:7-13. doi: 10.1016/j.jsr.2012.11.005. doi: 10.1016/j.jsr.2012.11.005

32. Raina,P., Sohel, N., Oremus, M., Shannon, H., Mony, P., Kumar, R.,... PURElnvestigators (2016). Assessing global risk factors for non-fatal injuries from road traffic accidents and falls in adults aged 35-70 years in 17 countries: a cross-sectional analysis of the Prospective Urban Rural Epidemiological (PURE) study. Injury Prevention, 22(2), 92-8. doi: 10.1136/injuryprev-2014-041476.

33. Singh R, Singh HK, Gupta SC, Kumar Y. Pattern, severity and circumtances of injuries sustained in road traffic accidents: a tertiary care hospital-based study. Indian J Community Med. 2014 Jan;39(1):30-4. doi: 10.4103/0970-0218.126353.

34. Stevens JA, Sogolow ED. Gender differences for non-fatal unintentional fall related injuries among older adults. Inj Prev. 2005 Apr;11(2):115-9. doi: 10.1136/ip.2004.005835.

35. El Tayeb S, Abdalla S, Mørkve O, Heuch I, Van den Bergh G. Injuries in Khartoum state, the Sudan: a household survey of incidence and risk factors. Int J Inj Contr Saf Promot. 2014;21(2):144-53. doi: 10.1080/17457300.2013.792283.

36. Krishnaswamy B, Gnanasambandam U. Falls in older people: national/ regional review of India. Chennai, Tamil Nadu, India: WHO background paper to the global report on falls among older persons; 2007

37. Zhang D, He Y, Liu M, Yang HB, Wu L, Wang JH, Yang SS, Chen J, Wang YY, Zeng J, Yao Y, Guo ML. [Study on incidence and risk factors of fall in the elderly in a rural community in Beijing]. Zhonghua Liu Xing Bing Xue Za Zhi. 2016 May;37(5):624-8. doi: 10.3760/cma.j.issn.0254-6450.2016.05.007.

38. Yiengprugsawan V, Stephan K, McClure R, Kelly M, Seubsman S, Bain C, Sleigh AC; Thai Cohort Study Team. Risk factors for injury in a national cohort of 87,134 Thai adults. Public Health. 2012 Jan;126(1):33-9. doi: 10.1016/j.puhe.2011.09.027.

39. Subramanian SV, Nandy S, Irving M, Gordon D, Lambert H, Davey Smith G. The mortality divide in India: the differential contributions of gender, caste, and standard of living across the life course. Am 
J Public Health. 2006 May;96(5):818-25. doi: 10.2105/AJPH.2004.060103.

40. Papalia, L., Goldoni, M., Spaggiari, M. C., Roscelli, F., Corradi, M., \& Mutti, A. (2012). Sleep disorders, risk of accidents and traffic accidents in a group of drivers of public transport. Giornale Italiano di Medicina del Lavoro ed Ergonomia, 34(3 Supplement), 353-356.

41. Brassington GS, King AC, Bliwise DL. Sleep problems as a risk factor for falls in a sample of community-dwelling adults aged 64-99 years. J Am Geriatr Soc. 2000 Oct;48(10):1234-40. doi: 10.1111/j.1532-5415.2000.tb02596.x.

42. Gureje O, Kola L, Ademola A, Olley BO. Profile, comorbidity and impact of insomnia in the Ibadan study of ageing. Int J Geriatr Psychiatry. 2009 Jul;24(7):686-93. doi: 10.1002/gps.2180.

43. Ebrahimi, M. H., Sadeghi, M., Dehghani, M., \& Niiat, K. S. (2015). Sleep habits and road traffic accident risk for Iranian occupational drivers. International Journal of Occupational Medicine and Environmental Health, 28(2), 305-132.

44. Gale CR, Cooper C, Aihie Sayer A. Prevalence and risk factors for falls in older men and women: The English Longitudinal Study of Ageing. Age Ageing. 2016 Nov;45(6):789-794. doi:

10.1093/ageing/afw129.

45. Korniloff K, Häkkinen A, Koponen HJ, Kautiainen H, Järvenpää S, Peltonen M, Mäntyselkä P, Kampman O, Oksa H, Vanhala M. Relationships between depressive symptoms and self-reported unintentional injuries: the cross-sectional population-based FIN-D2D survey. BMC Public Health. 2012 Jul 10;12:516. doi: 10.1186/1471-2458-12-516.

46. Yamashita T, Noe DA, Bailer AJ. Risk factors of falls in community-dwelling older adults: logistic regression tree analysis. Gerontologist. 2012 Dec;52(6):822-32. doi: 10.1093/geront/gns043.

47. Lukaszyk C, Harvey L, Sherrington C, Keay L, Tiedemann A, Coombes J, Clemson L, Ivers R. Risk factors, incidence, consequences and prevention strategies for falls and fall-injury within older indigenous populations: a systematic review. Aust N Z J Public Health. 2016 Dec;40(6):564-568. doi: 10.1111/1753-6405.12585.

48. Chang VC, Do MT. Risk factors for falls among seniors: implications of gender. Am J Epidemiol. 2015 Apr 1;181(7):521-31. doi: 10.1093/aje/kwu268.

49. Lord SR, Smith ST, Menant JC. Vision and falls in older people: risk factors and intervention strategies. Clin Geriatr Med. 2010 Nov;26(4):569-81. doi: 10.1016/j.cger.2010.06.002.

50. Stalenhoef PA, Diederiks JP, Knottnerus JA, Kester AD, Crebolder HF. A risk model for the prediction of recurrent falls in community-dwelling elderly: a prospective cohort study. J Clin Epidemiol. 2002 Nov;55(11):1088-94. doi: 10.1016/s0895-4356(02)00502-4.

51. Benegal V. India: alcohol and public health. Addiction. 2005;100(8):1051-6.

52. Li W, Keegan TH, Sternfeld B, Sidney S, Quesenberry CP Jr, Kelsey JL. Outdoor falls among middleaged and older adults: a neglected public health problem. Am J Public Health. 2006 Jul;96(7):1192200. doi: 10.2105/AJPH.2005.083055.

53. Ren J, Waclawczyk A, Hartfield D, Yu S, Kuang X, Zhang H, Alamgir H. Analysis of fall injuries by body mass index. South Med J. 2014 May;107(5):294-300. doi: 10.1097/SMJ.0000000000000097. 